\title{
The rapid prototyping of anatomic models in pulmonary atresia
}

\author{
Elizabeth M. Ngan, MD, MSc, ${ }^{a}$ Ivan M. Rebeyka, MD, FRCSC, ${ }^{b}$ David B. Ross, MD, FRCSC, ${ }^{\text {b }}$ \\ Mohamed Hirji, MB ChB, FRCPC, ${ }^{a}$ Johan F. Wolfaardt, BDS, MDent, PhD, ${ }^{\mathrm{c}, \mathrm{e}}$ Rosemary Seelaus, MAMS, ${ }^{\text {d,e }}$ \\ Andrew Grosvenor, MIMPT, LCGI, RDT, ${ }^{\mathrm{e}}$ and Michelle L. Noga, MD, FRCPC ${ }^{\mathrm{a}}$
}

Objective: The goal of this study was to assess the utility and accuracy of solid anatomic models constructed with rapid prototyping technology for surgical planning in patients with pulmonary atresia with ventricular septal defect and major aortopulmonary collateral arteries.

Methods: In 6 patients with pulmonary atresia with ventricular septal defect and major aortopulmonary collateral arteries, anatomic models of the pulmonary vasculature were rapid prototyped from computed tomographic angiographic data. The surgeons used the models for preoperative and intraoperative planning. The models' accuracy and utility were assessed with a postoperative questionnaire completed by the surgeons. An independent cardiac radiologist also assessed each model for accuracy of major aortopulmonary collateral artery origin, course, and caliber relative to conventional angiography.

Results: Of the major aortopulmonary collateral arteries identified during surgery and conventional angiography, $96 \%$ and $93 \%$, respectively, were accurately represented by the models. The surgeons found the models to be very useful in visualizing the vascular anatomy.

Conclusion: This study presents the novel vascular application of rapid prototyping to pediatric congenital heart disease. Anatomic models are an intuitive means of

From the Departments of Radiology and Diagnostic Imaging, ${ }^{a}$ Surgery, ${ }^{\text {b }}$ and Dentistry, ${ }^{\mathrm{c}}$ and the Faculty of Rehabilitation Medicine, ${ }^{\mathrm{d}}$ University of Alberta, Edmonton, Alberta, Canada, and COMPRU, Misericordia Community Hospital, Caritas Health Group, ${ }^{\mathrm{e}}$ Edmonton, Alberta, Canada.

Supported by a grant from the Caritas Health Group. Financial support for the Medical Modeling Research Laboratory was provided by the Government of Canada through Western Economic Diversification Canada.

Received for publication Sept 23, 2005; revisions received Dec 20, 2005; accepted for publication Feb 3, 2006.

Address for reprints: Elizabeth Ngan, MD, MSc, 2A2.41 Walter C. Mackenzie Health Sciences Centre, 8440-112 St, Edmonton, Alberta, Canada T6G 2B7 (E-mail: ema2@ ualberta.ca).

J Thorac Cardiovasc Surg 2006;132:264-9

$0022-5223 / \$ 32.00$

Copyright (๑) 2006 by The American Association for Thoracic Surgery

doi:10.1016/j.jtcvs.2006.02.047 communicating complex imaging data, such as the pulmonary vascular tree, which can be referenced intraoperatively.

$\mathrm{P}$ ulmonary atresia with ventricular septal defect (VSD) and major aortopulmonary collateral arteries (MAPCAs) is an uncommon form of cyanotic congenital heart disease in which the native pulmonary arteries are very hypoplastic or absent. To provide blood supply to the lungs, MAPCAs form during embryogenesis, probably from the splanchnic vascular plexus. ${ }^{1}$ These MAPCAs, which frequently have stenotic origins, arise from the aorta or one of its branches, and vary from patient to patient in number, origin, size, and course. ${ }^{2,3}$ For any given segment of lung, the blood supply may come from the native pulmonary artery, MAPCAs, or both. ${ }^{2}$

The goal of corrective surgery is to repair the intracardiac defects, establish right ventricular to pulmonary arterial continuity, and connect the MAPCAs to the pulmonary arterial system..$^{4-7}$ This process may require a single operation (singlestage unifocalization and complete intracardiac repair) or multiple operations (sequential unifocalization with subsequent intracardiac repair). ${ }^{4-7}$ One critical component of this surgery is the identification and isolation of the MAPCAs, a process made difficult by the patient-specific variations in anatomy. Adequate unifocalization of the MAPCAs is the key to successful surgery.

In more complex cases, the preoperative workup includes conventional angiography, computed tomographic (CT) angiography, or magnetic resonance (MR) 


$$
\begin{aligned}
& \text { Abbreviations and Acronyms } \\
& \begin{aligned}
\text { 3D } & =3 \text {-dimensional } \\
\mathrm{CT} & =\text { computed tomography } \\
\mathrm{MAPCA} & =\text { major aortopulmonary collateral artery } \\
\mathrm{MR} & =\text { magnetic resonance } \\
\mathrm{VSD} & =\text { ventricular septal defect }
\end{aligned}
\end{aligned}
$$

angiography to map out the pulmonary vascular supply. ${ }^{8-12}$ The precise 3-dimensional (3D) relationship of MAPCAs to the aorta and native pulmonary arteries is significant and should be rendered into a useful, intuitively graspable format that is easily referenced intraoperatively. Rapid prototyping provides a potential solution to this problem.

Rapid prototyping is the fabrication of solid models from computer-generated virtual 3D surface models. The virtual model is first broken down into thin slices or layers. A rapid prototyping machine then builds the solid model layer upon layer, resulting in a physical replica of the virtual model. ${ }^{13}$ In medical applications of this technology, the virtual models are typically constructed from CT or MR imaging data sets.

The most extensive medical applications of rapid prototyping technology are in head and neck, oral, and craniomaxillofacial surgery. ${ }^{14-16}$ Models of the bony anatomy of the head and neck are used to help guide surgery and reconstruction. Relatively limited numbers of vascular applications of this technology have also been developed. These vascular applications include models of heart valves, ${ }^{17-20}$ aortic aneurysms, ${ }^{21}$ intracranial aneurysms, ${ }^{22}$ carotid arteries, ${ }^{23}$ and embryonic hearts. ${ }^{24,25}$

\section{Methods}

\section{Patient Selection}

Six consecutive patients, aged 6 months to 2 years 6 months at the time of surgery, with pulmonary atresia with VSD and MAPCAs presenting to the Stollery Children's Hospital in Edmonton, Alberta, Canada were studied. The operations took place between May 2004 and June 2005. Additional patients in whom the imaging was not performed sufficiently in advance of the surgery to allow model construction were excluded from the study. This study was approved by the Health Research Ethics Board at the University of Alberta Hospital. A parent or guardian of each patient gave informed consent.

\section{Imaging Studies}

All patients underwent both conventional angiography and CT angiography of the aorta and pulmonary arterial vasculature. Because of the complexity of this type of congenital anomaly, CT angiography was considered part of the standard preoperative evaluation. No additional CT angiography was performed for the purpose of this study. The CT angiograms were obtained with $0.625-\mathrm{mm}$ or $1.25-\mathrm{mm}$ thickness slices with $0.625-\mathrm{mm}$ reconstruction and a slice overlap of $50 \%$ on a 16 slice General Electric Light Speed CT scanner (GE Medical Systems, Milwaukee, Wis). The patients were under general anesthesia, and positive-pressure breath-hold technique was used during image acquisition. Intravenous contrast was administered at a dose of $2 \mathrm{~mL} / \mathrm{kg}$ over 10 to 15 seconds. The patients were 4 months 6 days to 2 years 4 months old at the time of CT angiography, with 5 of the 6 patients 6 months old or younger. The CT was performed at least 2 weeks before the operation, which allowed time for the model to be built.

\section{Model Construction}

The models of the CT data sets were created with Mimics 8.11 software (Materialise, Leuven, Belgium) and a rapid prototyping machine. In Mimics, a virtual 3D model was created of the pulmonary arterial vasculature and aorta by a radiology resident and a pediatric cardiac radiologist. Smoothing of the model was performed with Magics 8.01 software (Materialise) or haptic modeling software and hardware (FreeForm Modeling System, version 7.0 and PHANTOM Desktop Haptic Device; SensAble Technologies, Woburn, Mass). This virtual model was converted into a solid acrylic or plastic anatomic model with a rapid prototyping machine, either the Stratasys Prodigy Plus (Stratasys Inc, Eden Prairie, Minn) or the InVision si2 3-D printer (3D Systems, Valencia, Calif). The image manipulation and rapid prototyping were carried out in the Medical Modeling Research Laboratory, COMPRU, Caritas Health Group, Edmonton, Alberta, Canada. The models were sterilized and used within the operative field at the time of surgery.

To study the accuracy of this construction technique, several test objects containing both flat and cylindric surfaces were imaged and then modeled in the same manner as the patient data. The objects and the models were then measured with digital calipers (Absolute Digimatic calipers; Mitutoyo, MTI Canada Ltd, Mississauga, Ontario, Canada).

\section{Model Evaluation}

The assessment of the models was 2-fold. First, after each operation, the surgeons completed a short questionnaire to rate the model's overall usefulness and accuracy. The surgeons rated utility and accuracy on 3-point scales (very useful, somewhat useful, or not useful, and very accurate, minor inaccuracies, or major inaccuracies, respectively). MAPCAs identified at the time of surgery were also quantified. Each MAPCA on the models was also rated on a 3-point scale (accurate, minor inaccuracy, or inaccurate).

Second, an independent pediatric cardiac radiologist compared the models with the angiograms. The cardiac radiologist compared the number of MAPCAs on the model with the number demonstrated on the corresponding angiogram. The accuracy of the origin, course, and caliber of each MAPCA on the model was rated on a 3-point scale (accurate, minor inaccuracy, or major inaccuracy). Minor inaccuracy was defined as acceptable accuracy with a minimal and clinically irrelevant discrepancy between the model and angiogram. Major inaccuracy was defined as unacceptable accuracy, potentially misleading to the surgeon and not reflecting the angiographic findings. The models were therefore compared to the absolute standard, surgery, and to the imaging standard, conventional angiography.

The funding organizations, Caritas Health Group and the Government of Canada through Western Economic Diversification Canada, had no role in the study design; in data collection, analysis, and interpretation; in writing this report; or in the decision to publish the results. 

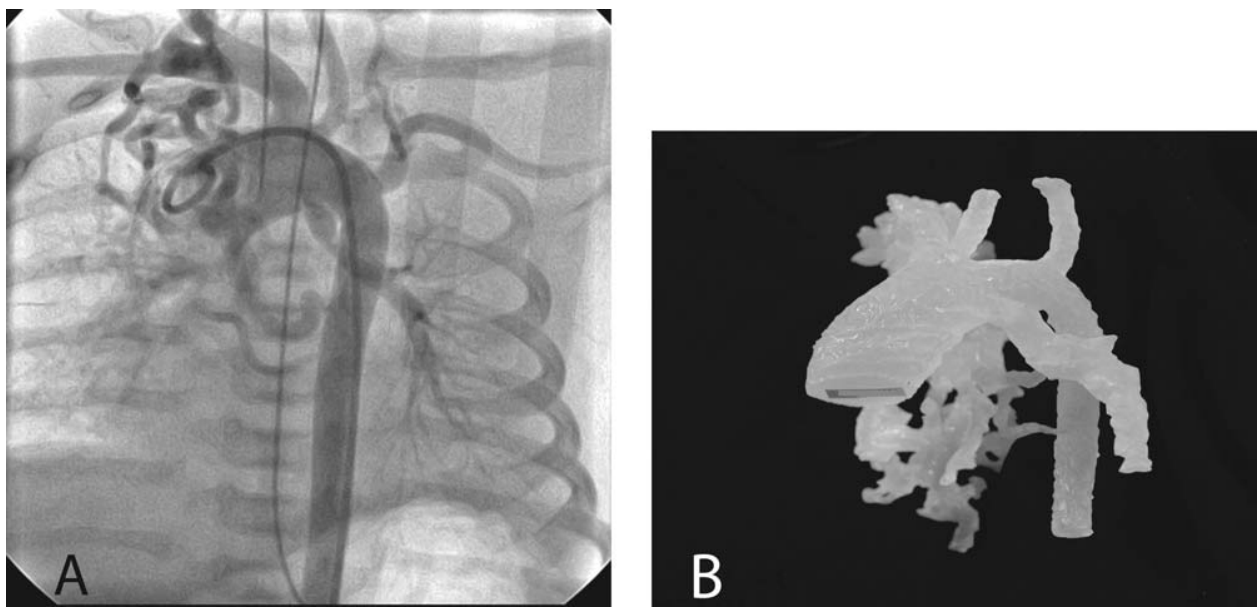

Figure 1. Comparison of angiogram (A) with model (B) of thoracic aorta and MAPCAs in patient with pulmonary atresia, native left pulmonary artery, and MAPCAs (case 4).

\section{Results}

Six patients were studied, with intraoperative results available in 5 cases. In case 4, the patient was referred to another center for surgery because of an earlier available operating date, so no intraoperative results are available. Figures 1 and 2 are photographs of models with the accompanying angiographic images.

Figure 3 and Table 1 compare the numbers of MAPCAs identified with each of the modalities: model based on CT angiography, conventional angiography, and intraoperative results. Of 25 MAPCAs identified at the time of surgery, 24 (96\%) were accurately represented by the models. Of 30 MAPCAs identified on angiography, 28 (93\%) were also accurately depicted on the models. One MAPCA was identified during surgery and on the angiogram but not on the model (case 3). This small (approximately $3 \mathrm{~mm}$ diameter) vessel was not identified as a MAPCA on the CT data because of its small caliber, its close proximity to the true pulmonary arteries, and table motion artifact affecting this particular CT study. This case demonstrates the limitations of CT angiography. Conversely, 1 MAPCA identified at surgery was found on the CT study and model but not the angiogram (case 2). This vessel was probably not identified by conventional angiography because of overlapping vessels on the initial two projections obtained, so selective catheterization of this MAPCA was not pursued. In addition, 1 MAPCA less than approximately $2 \mathrm{~mm}$ in diameter identified on angiography was not identifiable even in retrospect from the CT angiographic data (case 4). This vessel may have become occluded between the time of the angiography and the time of the CT (5 months 7 days), or its caliber may have been below the threshold of CT. In this case, intraoperative results are not available for confirmation of either interpretation. In this limited series, the models based on CT angiography rivaled conventional angiography in the identification of MAPCAs.

The surgeons' assessments of the overall utility and accuracy of the models are presented in Figure 4. The
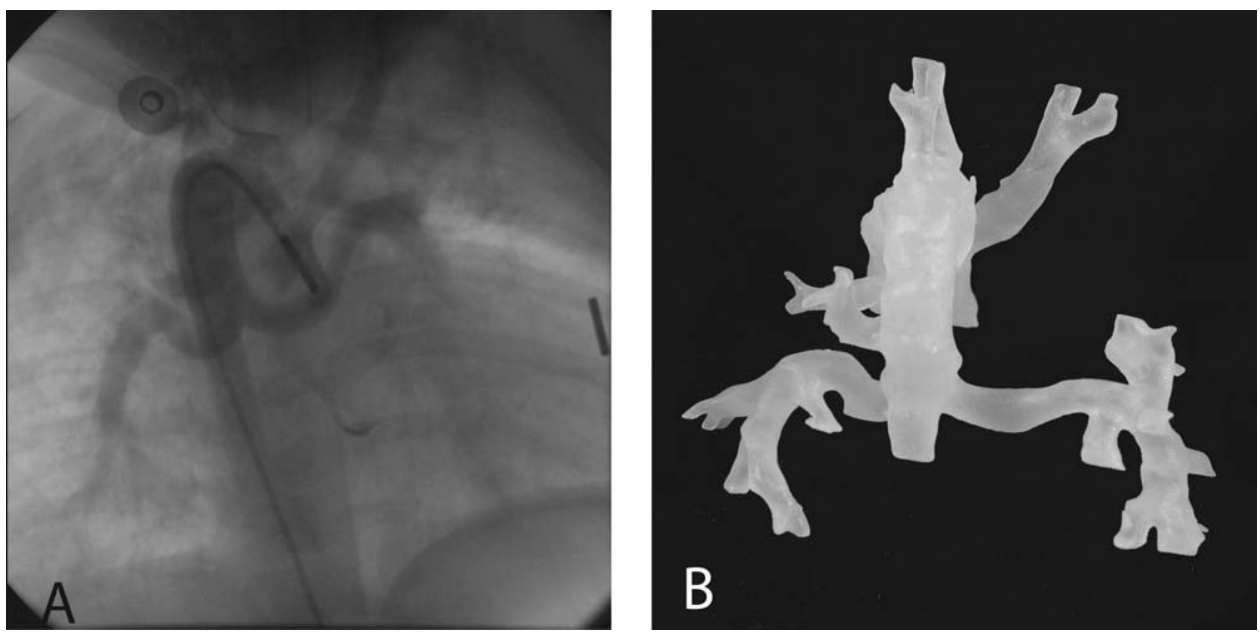

Figure 2. Comparison of angiogram $(A)$ with model $(B)$ of thoracic aorta and MAPCAs in patient with pulmonary atresia and MAPCAs (case 1). 


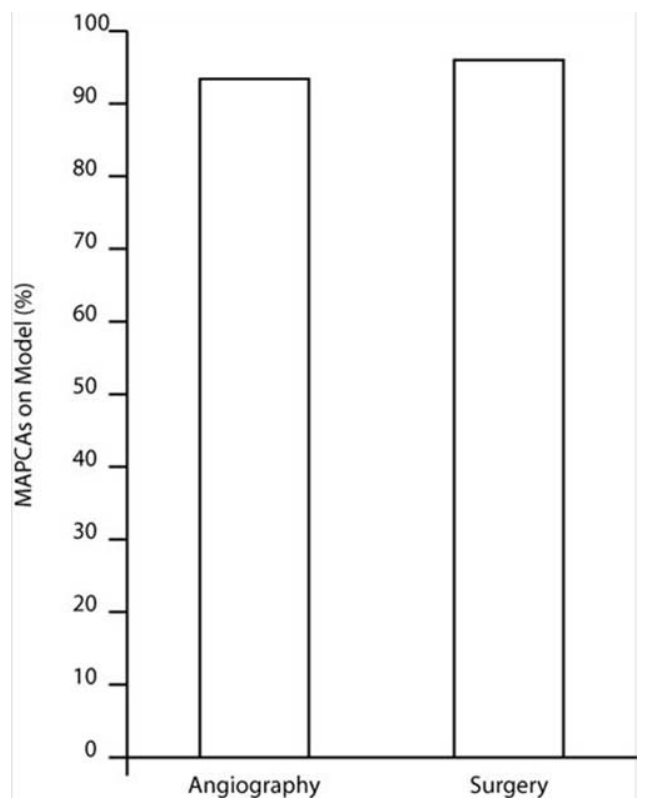

Figure 3. Percentage of MAPCAs identified on angiograms and during surgery included on models.

models were all deemed to be very useful, and most were considered very accurate. The only model not receiving a very accurate rating was the model in which 1 of the MAPCAs was not identified. Each MAPCA was also rated by the surgeons for overall accuracy of position. Only the previously mentioned unidentified MAPCA was not rated by the surgeons as accurately representing the intraoperative findings. The subjective impressions of one of the surgeons were that the models aided in surgical planning, allowed rapid isolation of the MAPCAs, and decreased operating room time.

The initial impression of one of the surgeons was that the models were somewhat smaller than the actual blood vessels. To confirm the accuracy of our modeling techniques, several cylindric test objects were scanned and modeled.

TABLE 1. Identification of MAPCAs in the model, on angiography, and at the time of surgery

\begin{tabular}{cccc}
\hline Case & $\begin{array}{c}\text { MAPCAs in } \\
\text { model }\end{array}$ & $\begin{array}{c}\text { MAPCAs on } \\
\text { angiogram }\end{array}$ & $\begin{array}{c}\text { MAPCAs during } \\
\text { surgery }\end{array}$ \\
\hline 1 & 4 & 4 & 4 \\
2 & 4 & 3 & 4 \\
3 & 5 & 6 & 6 \\
4 & 5 & 6 & $\mathrm{NA}$ \\
5 & 5 & 5 & 5 \\
6 & 6 & 6 & 6 \\
Total & 29 & 30 & 25
\end{tabular}

NA, Not applicable.
The test models all had diameters slightly larger than the original objects, from $0.26 \mathrm{~mm}(2.0 \%)$ to $0.85 \mathrm{~mm}(13.2 \%)$ larger. In no case was the model's diameter smaller than that of the original. The apparent size discrepancy between the blood vessels and the model is thus unlikely to be due to the technique used to build the model. Other possible explanations include the growth of the child in the interval between the CT and the surgery (as long as 4 months 29 days) and the fact that the model is of the vascular space within the blood vessel, not the external surface of the vessel as viewed by the surgeon.

A total of 31 MAPCAs were identified on the models or on the angiograms. Figure 5 summarizes the findings of the independent pediatric cardiac radiologist who compared each of the models with the angiograms, rating the origin, course, and caliber of each model MAPCA for accuracy. Of the MAPCA origins, 93\% were rated as having acceptable accuracy, and $97 \%$ of the MAPCAs received an acceptable accuracy rating for course and caliber. The MAPCA in case 4 not identifiable on CT was rated as having unacceptable accuracy in all categories. The MAPCA in case 3 that was not identified as a MAPCA but rather included on the model as a branch of the right pulmonary artery received an inaccurate origin rating. Only 2 MAPCAs were rated as having minor inaccuracies. In both cases, the inaccuracy was in the caliber of a stenosis. Although it was not part of the questionnaire completed by the cardiac radiologist, he also noted several cases in which the models were superior to the angiograms. The most obvious example of this is the MAPCA that was not identified on the angiogram but was accurately included on the model, matching the operative findings (case 2).

\section{Discussion}

This study provides an initial assessment of the application of rapid prototyping technology to pediatric congen-

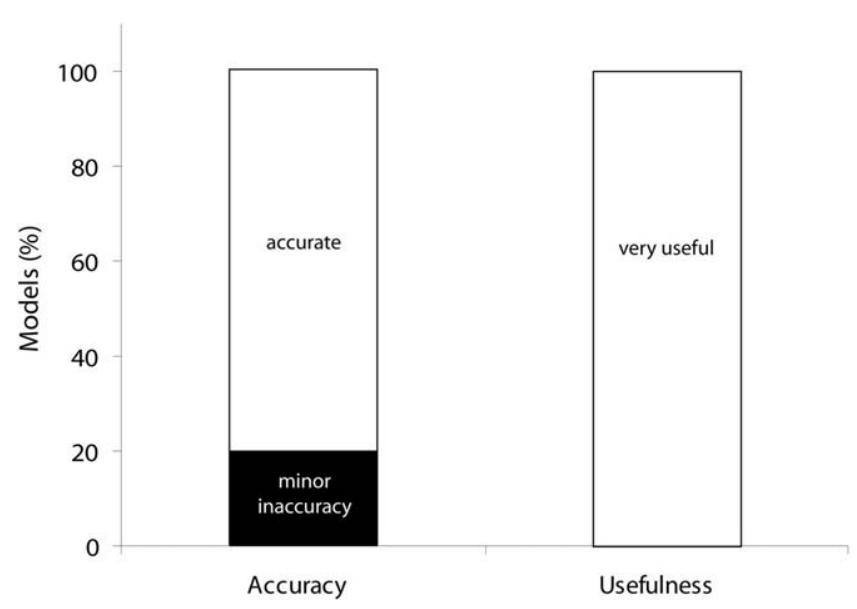

Figure 4. Surgeons' ratings of model utility and accuracy. 


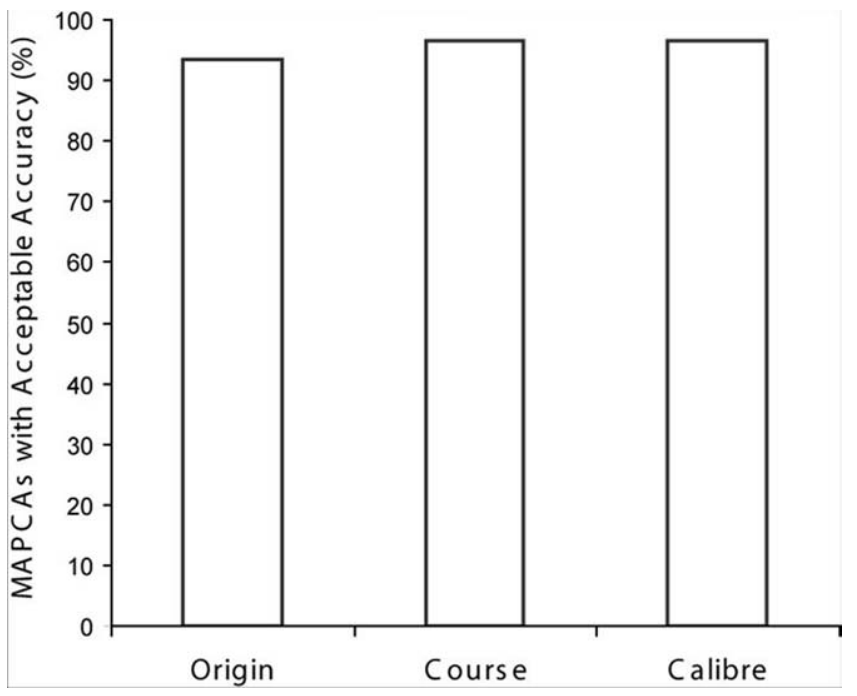

Figure 5. Cardiac radiologist's ratings of model accuracy relative to conventional angiography.

ital heart disease, specifically pulmonary atresia with VSD and MAPCAs. Previous imaging studies of pulmonary atresia with VSD and MAPCAs focused on conventional imaging modalities such as angiography, CT angiography, and MR angiography. ${ }^{8-12}$ Rapid prototyping technology takes imaging data a step beyond 2-dimensional computer displays to solid anatomic models. This study adds to the limited number of reported soft tissue and vascular applications of this technology.

\section{Technical Considerations}

Models of soft tissue structures are more difficult to construct than models of bony structures because of the much smaller variation in Hounsfield units, which is significant. Obviously, contrast administration greatly helps in modeling vascular structures. In this particular application, careful attention to anatomy is required to separate the pulmonary arteries from the pulmonary veins. As a result, virtual model construction cannot be automated and should be performed by a clinical specialist. One technical limitation of the models is their fragility, particularly at stenoses. As a result, the models must be handled with care. The techniques presented in this article represent a starting point in optimizing the model construction of congenital heart disease.

\section{Clinical Implications}

Of MAPCAs identified during surgery and conventional angiography, $96 \%$ and $93 \%$, respectively, were accurately represented by the models. These values are in line with previous accuracy reports of CT angiography and MR angiography of this condition. ${ }^{11,12}$ The accuracy of the models is limited by the data set on which they are based, CT angiography. Two MAPCAs found either during surgery or by conventional angiography were not included on the models. In both cases, when the CT data were retrospectively assessed, it was either impossible or difficult to identify the missed MAPCAs. Contributing factors to these MAPCAs being missed were degraded images from table motion artifact and probable occlusion of a small vessel in the interval between the conventional angiogram and the CT angiogram. However, 1 MAPCA identified on the CT study and so included on the model was missed at angiography. This demonstrates the complementary nature of CT angiography with rapid prototyping and conventional angiography in identifying as many MAPCAs as possible before surgery. Because of the need for maximum accuracy of imaging, we believe that CT angiography with rapid prototyping provides useful supplemental information in addition to conventional angiography. In this study, all MAPCAs were identified either on the CT or the angiogram before surgery.

However, simple identification of vessels is not adequate. This study extends the utility of CT angiography by presenting the data in a more useful format, a solid anatomic model showing the course, caliber, and origin of each MAPCA. Solid models communicate information in both visual and tactile formats; however, it is difficult to quantitatively evaluate the conveyance of information, particularly tactile information. The surgeons' overall impression of the ability of the anatomic models to communicate the necessary spatial information was quantified as usefulness in this study. The surgeons rated all the models as very useful for preoperative and intraoperative planning, allowing rapid isolation of MAPCAs during the surgery. One surgeon's impression was that the models decreased operating room time, although this study did not measure operating room time as an outcome variable.

The surgeons had available to them a virtual model on a workstation preoperatively and the solid model both preoperatively and intraoperatively. We view the models as an addition to rather than a replacement for a conventional workstation display. On the basis of this limited experience, the 2-dimensional workstation screens did not convey the information as intuitively as the solid models.

\section{Study Limitations}

The number of patients is one of the limiting factors in this study. In a larger study, variables such as operating room time and patient outcome could be examined. Because of the small number of patients, accounting for anatomic variations and comorbidities that affect prognosis would be difficult. Because pulmonary atresia with VSD and MAPCAs is a relatively rare condition, time is required to assess a larger number of patients. The creation of virtual models was relatively labor intensive and required expert knowledge of the pulmonary vasculature. Automated virtual model 
construction was not possible with the current software used (Mimics).

\section{Conclusion}

This study represents a starting point in the investigation of rapid prototyping technology as it applies to complex pediatric congenital heart disease. The 3D anatomic models produced were found to be useful in operative planning for pulmonary atresia with MAPCAs. Further optimization of the imaging and virtual model construction techniques is planned. In addition, this technology has the potential to aid in the preoperative assessment of a broader range of conditions in congenital heart disease. The utility and application of rapid prototyping in other forms of congenital heart disease need to be assessed.

\section{References}

1. Liao PK, Edwards WD, Julsrud PR, Puga FJ, Danielson GK, Feldt RH. Pulmonary blood supply in patients with pulmonary atresia and ventricular septal defect. J Am Coll Cardiol. 1985;6:1343-50.

2. Haworth SG, Macartney FJ. Growth and development of pulmonary circulation in pulmonary atresia with ventricular septal defect and major aortopulmonary collateral arteries. Br Heart J. 1980;44:14-24.

3. Jefferson K, Rees S, Somerville J. Systemic arterial supply to the lungs in pulmonary atresia and its relation to pulmonary artery development. Br Heart J. 1972;34:418-27.

4. Lofland GK. The management of pulmonary atresia, ventricular septal defect and multiple aorto pulmonary collateral arteries by definitive single stage repair in early infancy. Eur J Cardiothorac Surg. 2000; 18:480-6.

5. Puga FJ, Leoni FE, Julsrud PR, Mair PD. Complete repair of pulmonary atresia, ventricular septal defect and severe peripheral arborization abnormalities of the central pulmonary arteries. Experience with preliminary unifocalization procedures in 38 patients. $J$ Thorac Cardiovasc Surg. 1989;98:1018-29.

6. Gupta A, Odim J, Levi D, Chang RY, Laks H. Staged repair of pulmonary atresia with ventricular septal defect and major aortopulmonary collateral arteries: experience with 104 patients. J Thorac Cardiovasc Surg. 2003;126:1746-52.

7. Reddy VM, Liddicoat JR, Hanley FL. Midline one-stage complete unifocalization and repair of pulmonary atresia with ventricular septal defect and major aortopulmonary collaterals. J Thorac Cardiovasc Surg. 1995;109:832-44.

8. Okada M, Matsunaga N, Ito K, Takano K, Fujiwara M, Ueda K. Modified Blalock-Taussig shunt patency for pulmonary atresia: assessment with electron beam CT. J Comput Assist Tomogr. 2002;26:368-72.

9. Westra SJ, Hurteau J, Galindo A, McNitt-Gray MF, Boechat MI, Laks H. Cardiac electron-beam CT in children undergoing surgical repair for pulmonary atresia. Radiology. 1999;213:502-12.
10. Rebergen SA, deRoos A. Congenital heart disease. Evaluation of anatomy and function by MRI. Herz. 2000;25:365-83.

11. Murai S, Hamada S, Yamamoto S, Khankan AA, Sumikawa H, Inoue A, et al. Evaluation of major aortopulmonary collateral arteries (MAPCAs) using three-dimensional CT angiography: two case reports. Radiat Med. 2004;22:186-9.

12. Geva T, Greil GF, Marshall AC, Landzberg M, Powell AJ. Gadoliniumenhanced 3-dimensional magnetic resonance angiography of pulmonary blood supply in patients with complex pulmonary stenosis or atresia: comparison with x-ray angiography. Circulation. 2002;106: 473-8.

13. Webb PA. A review of rapid prototyping (RP) techniques in the medical and biomedical sector. J Med Eng Technol. 2000;24:149-53.

14. D'Urso PS, Barker TM, Earwaker WJ, Bruce LJ, Atkinson RL, Lanigan MW, et al. Stereolithographic biomodelling in cranio-maxillofacial surgery: a prospective trial. J Craniomaxillofac Surg. 1999;27:30-7.

15. Park GC, Wiseman JB, Clark WD. Correction of congenital microtia using stereolithography for surgical planning. Plast Reconstr Surg. 2000;105:1444-7.

16. Chang PS, Parker TH, Patrick CW Jr, Miller MJ. The accuracy of stereolithography in planning craniofacial bone replacement. J Craniofac Surg. 2003;14:164-70.

17. Gilon D, Cape EG, Hanschumacher MD, Jiang L, Sears C, Solheim J, et al. Insights from three-dimensional echocardiographic laser stereolithography: effect of leaflet funnel geometry on the coefficient of orifice contraction, pressure loss, and the Gorlin formula in mitral stenosis. Circulation. 1996;94:452-9.

18. Sodian R, Loebe M, Hein A, Martin DP, Hoerstrup SP, Potapov EV, et al. Application of stereolithography for scaffold fabrication for tissue engineered heart values. ASAIO J. 2002;48:12-6.

19. Gilon D, Cape EG, Handschumacher MD, Song JK, Solheim J, VanAuker M, et al. Effect of three-dimensional valve shape on the hemodynamics of aortic stenosis: three-dimensional echocardiographic stereolithography and patient studies. J Am Coll Cardiol. 2002;40: 1479-86.

20. Binder TM, Moertl D, Mundigler G, Rehak G, Franke M, Delle-Karth $\mathrm{G}$, et al. Stereolithographic biomodeling to create tangible hard copies of cardiac structures from echocardiographic data: in vitro and in vivo validation. J Am Coll Cardiol. 2000;35:230-7.

21. Kato K, Ishiguchi T, Maruyama K, Naganawa S, Ishigaki T. Accuracy of plastic replica of aortic aneurysm using 3D-CT data for transluminal stent-grafting: experimental and clinical evaluation. J Comput Assist Tomogr. 2001;25:300-4.

22. Tateshima S, Murayama Y, Villablanca JP, Morino T, Takahashi H, Yamauchi $\mathrm{T}$, et al. Intraaneurysmal flow dynamics study featuring an acrylic aneurysm model manufactured using a computerized tomography angiogram as a mold. J Neurosurg. 2001;95:1020-7.

23. Yedavalli RV, Loth F, Yardimci A, Pritchard WF, Oshinski JN, Sadler $\mathrm{L}$, et al. Construction of a physical model of the human carotid artery based upon in vivo magnetic resonance images. J Biomech Eng. 2001;123:372-6.

24. Pentecost JO, Icardo J, Thornburg KL. 3D computer modeling of human cardiogenesis. Comput Med Imaging Graph. 1999;23:45-9.

25. Pentecost JO, Sahn DJ, Thornburg BL, Gharib M, Baptista A, Thornburg KL. Graphical and stereolithographic models of the developing human heart lumen. Comput Med Imaging Graph. 2001;25:459-63. 